\title{
Hyperarousal and sleep reactivity in insomnia: current insights
}

This article was published in the following Dove Press journal:

Nature and Science of Sleep

\author{
David A Kalmbach' \\ Andrea S \\ Cuamatzi-Castelan' \\ Christine V Tonnu' \\ Kieulinh Michelle Tran' \\ Jason R Anderson ${ }^{2}$ \\ Thomas Roth' \\ Christopher L Drake' \\ 'Thomas Roth Sleep Disorders \\ and Research Center, Henry Ford \\ Health System, Detroit, MI, USA; \\ ${ }^{2}$ Department of Psychological \\ Sciences, Kent State University, \\ Kent, OH, USA
}

\begin{abstract}
Hyperarousal is a key component in all modern etiological models of insomnia disorder. Overall patterns in the literature suggest that over-active neurobiological and psychological systems contribute to difficulty sleeping. Even so, mixed results regarding the specific mechanisms linking hyperarousal to sleep disturbance limit current etiological conceptualizations. Similar basal arousal profiles between individuals with high vs low risk for insomnia in the absence of stress exposure suggest that dysregulated stress "response" rather than general hyperarousal may be a more pertinent marker of risk. In this report, we discuss evidence for hyperarousal in insomnia and explore the role of sleep reactivity. A trait characteristic, sleep reactivity is the degree to which stress disrupts sleep, manifesting as difficulty falling and staying asleep. Premorbid sleep reactivity has been shown to identify individuals at risk for future insomnia disorder, such as highly reactive sleepers (whose sleep systems are sensitive to stress) who are at elevated disease risk. Research points to genetics, family history of insomnia, gender, and environmental stress as factors that influence sleep reactivity. Importantly, stress-related cognitive-emotional reactivity (e.g., rumination, worry) may exploit the vulnerability of a highly reactive sleep system. We propose that sleep reactivity and cognitive-emotional reactivity may share a bidirectional relationship, conferring an insalubrious environment for sleep in response to stress. Future research on sleep reactivity is needed to identify its neurobiology, characterize its relationship with cognitive-emotional reactivity, and explore the potential clinical utility of sleep reactivity in treatment planning.
\end{abstract}

Keywords: insomnia, hyperarousal, stress, sleep reactivity

\section{Introduction}

Insomnia is the most common sleep disorder in the US with prevalence rates estimated between $9 \%$ and $20 \%$ among adults. ${ }^{1,2}$ Despite advances in pharmacological and behavioral interventions, ${ }^{3-8}$ many treated insomniacs relapse ${ }^{9}$ and those with severe forms of insomnia are resistant to treatment. ${ }^{10}$ Understanding the course of insomnia is difficult, in part, because its complex psychoneurobiology is not yet fully understood. However, recent advances using hyperarousal and stress dysregulation models of insomnia have offered critical insights into insomnia disease processes.

The concept of hyperarousal as an important etiological factor in insomnia has been long held, with serious scientific inquiry into this conceptualization, as well as the issue of cause and effect, undertaken more than 50 years ago. Several important factors have led to an increasing interest in this broadly defined area of sleep science. One factor is the near exponential rise of sleep science over the last 2 decades (Figure 1).
Correspondence: Christopher L Drake Sleep Research Laboratory, Thomas Roth Sleep Disorders and Research Center, Henry Ford Health System, 39450 W 12 Mile Road, Novi, MI 48377, USA

Tel +l 2483446672

Fax + I 2483448084

Email cdrakel@hfhs.org 


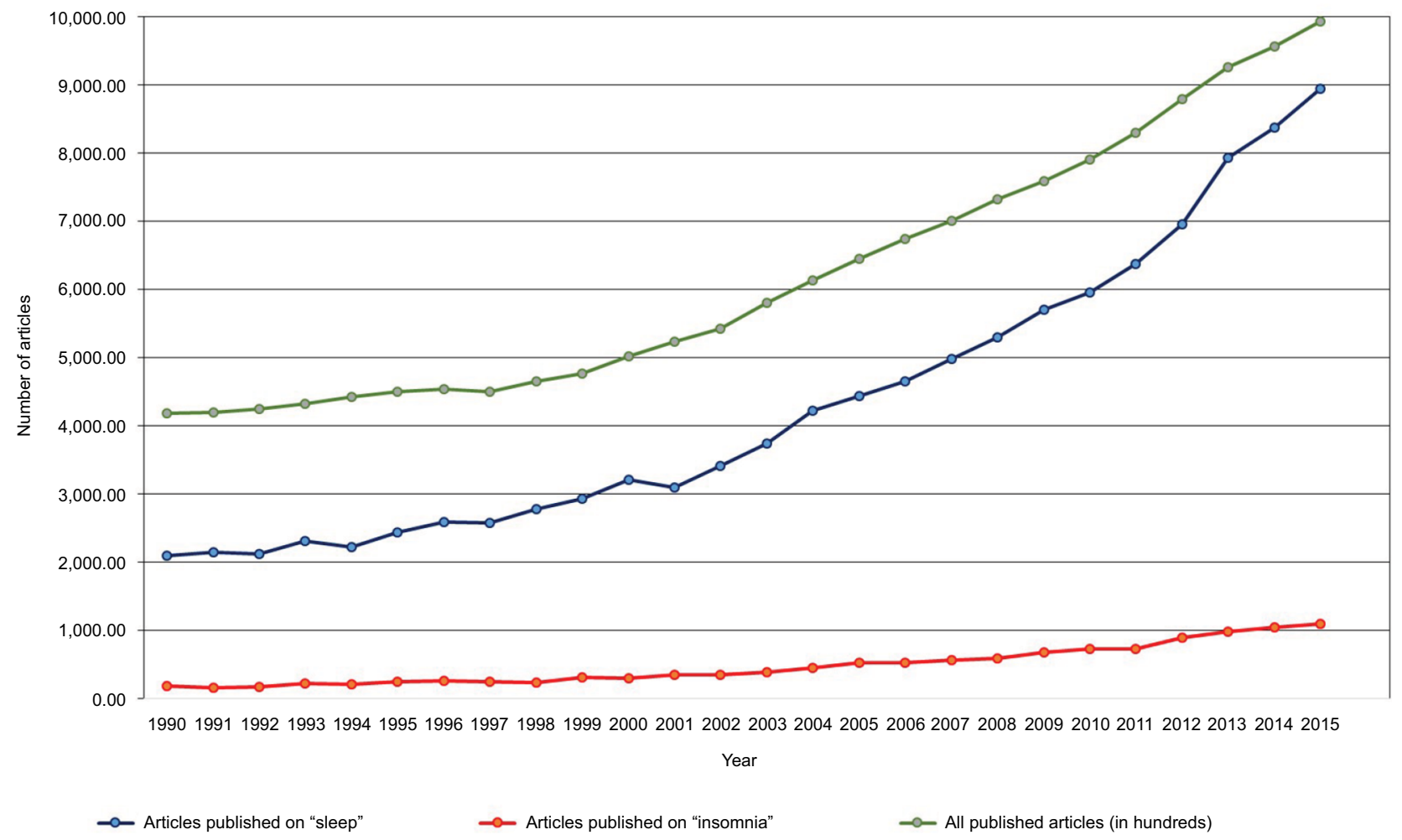

Figure I All published articles including keywords "sleep" and "insomnia" from 1990 to 2015. Note: Data were obtained from Ovid-MEDLINE database.

Another contributing factor is the now wide availability and low cost of tools to study aspects of hyperarousal including its physiological, cognitive, and neuronal correlates.

A critical challenge for our current understanding of hyperarousal in insomnia is to synthesize these oftendisparate findings both across and within these broad domains. Similar to terms such as stress, reactivity, and other broad constructs that cross the boundaries of psychology, physiology, and medicine, the term "hyperarousal" is difficult, if not impossible, to define without sufficient context. Indeed, many conceptualizations exist that vary not only between but also within disciplines. Thus, there has been an expansion in the scientific study of all aspects of sleep-related hyperarousal becoming practically as broad as the concept itself. While this expansion both across and within domains has led to numerous important findings, it has also produced an ever-widening gap between researchers who are essentially studying the same construct.

There have been many excellent and encompassing reviews of hyperarousal in insomnia since 2000 (see studies by Riemann, ${ }^{11}$ Bonnet and Arand, ${ }^{12}$ Roth et al, ${ }^{13}$ Harvey et al,${ }^{14}$ Kay and Buysse, ${ }^{15}$ Drummond et al, ${ }^{16}$ and Vgontzas et al, ${ }^{17}$ among others); therefore, we will attempt to not retread here. Some have focused on physiological components, some have provided new insights regarding neuroimaging and cognitive findings, and others have explored the role of hyperarousal in insomnia phenotyping. In the current review, we will begin by briefly considering the literature on hyperarousal in insomnia, and then shift focus to explore a recent and quickly emerging area of insomnia research that is proving to be an integral component of the hyperarousal model of insomnia: sleep reactivity.

\section{Hyperarousal and stress dysregulation in insomnia}

The many studies of physiological measures associated with insomnia disorder point to general signs of hyperarousal in insomnia, but with important caveats and inconsistencies. For example, although stressful life events have long been shown to be important triggers of insomnia, ${ }^{18-20}$ traditional physiological markers of stress and arousal such as hypothalamic-pituitary-adrenal (HPA) axis (e.g., cortisol, adrenocorticotropic hormone) and sympathetic nervous system (SNS) activation have produced varied findings over the past 
3 decades of insomnia research. ${ }^{21-28}$ Neuroimaging studies of insomnia also show disparate findings with respect to any concept of "general hyperarousal" with several studies suggesting higher activation in insomnia, ${ }^{29-31}$ although some evidence suggests hypoarousal. ${ }^{32} \mathrm{~A}$ recent review of this literature emphasized dysregulation in separate components of wake drive, sleep drive, and conscious awareness that likely contribute to these seeming inconsistencies. ${ }^{15}$ The idea that wake drive may be dysregulated independent of sleep drive (and vice versa) and that independent alterations within these systems may differentially impact nighttime insomnia symptoms (eg, sleep complaints) highlights a complexity that has continued to challenge the scientific understanding of hyperarousal in insomnia.

As in most areas of science, findings in insomnia have produced a more complex array of questions. What is the underlying neurophysiology that accounts for insomnia symptoms? If it is increased neurophysiological brain activation (ie, central nervous system hyperarousal), what areas of brain are critical for the perception of poor sleep? And do those brain $\operatorname{areas}^{29}$ or specific pathways ${ }^{33}$ also account for polysomnography (PSG)-defined insomnia-related sleep disturbances (ie, objective insomnia)?

One approach to clarifying some of the complexities in understanding the role of hyperarousal in insomnia has been to study the neurophysiological and cognitive processes in individuals at risk for insomnia prior to the development of chronic sleep symptoms (ie, before insomnia develops). This approach has been used to disentangle potential causes from consequences of insomnia and has yielded several important findings along with additional questions over the past decade. For example, recent findings have shown undamaged SNS functioning both basally and in response to standardized cognitive and physiological stress challenges along with concomitant dysregulation in HPA axis reactivity in insomniacs and individuals at elevated risk for insomnia. ${ }^{26,27}$ Interestingly, despite a dysregulated HPA axis response in individuals at risk for insomnia, no differences in the cognitive response to the laboratory stress were present, challenging the notion that the critical vulnerability to insomnia is a purely a cognitive phenomenon. ${ }^{27}$ Indeed, previous studies show that normal sleeping individuals at risk for insomnia also have a differential sleep alteration following predominantly physical sleep challenges. Do these findings reflect hyperarousal as a potential etiological risk factor for the development of symptoms in insomnia disorder? The absence of differences in basal measures of hyperarousal (cortisol, alpha amylase, heart rate, blood pressure) prior to stressful challenges suggests that dysregulated stress "response" rather than general hyperarousal may be a critical marker of risk.

Stress response is a broad concept that encompasses cognitive, emotional, and neurobiological reactions. Stress exposure is equally myriad, varying by source (eg, mental stress, physiologic pain, circadian challenge, substance consumption), potency (mild to severe), and duration or schedule (acute, chronic, intermittent, and so on). Not only do stressors vary greatly, but also individuals vary greatly in their response to stressors. That is, different individuals react differently even to uniform stressors, thus emphasizing the complex machinations of stress regulation. ${ }^{34-36}$ And it is precisely these differences in stress dysregulation that contribute to interindividual differences in risk for insomnia. ${ }^{35-37}$

Evidence suggests that insomniacs exhibit exaggerated and maladaptive neurobiological and cognitive-emotional reactivity to stress. ${ }^{11,19,38-41}$ However, it is unclear whether this stress dysregulation has a sleep-specific component (Figure 2). And while growing evidence suggests that stress dysregulation typically preexists and even contributes to insomnia development, it had been unclear until recently whether this stress dysregulation produces a uniquely sleepspecific vulnerability or rather a broad cognitive-emotional response that can impact a myriad of stress-linked physiological systems, such as sleep regulation, HPA axis, and autonomic nervous system (ANS). In recent years, research has identified that a sleep-specific component of stress reactivity exists, and the degree to which stress reactivity manifests in the sleep system has been referred to as situational insomnia ${ }^{42}$ and, now more commonly, "sleep reactivity". ${ }^{88}$

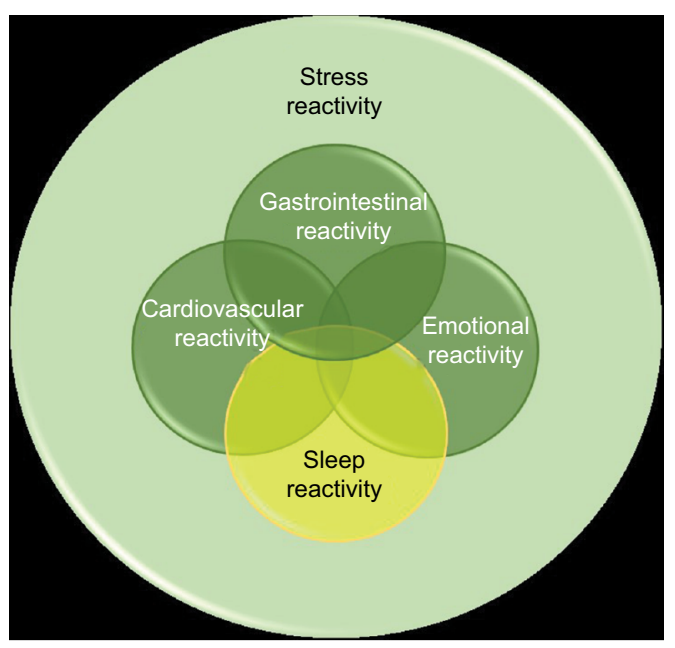

Figure 2 Diagram indicating the hypothesized relationship between sleep reactivity and the other components of stress reactivity.

Note: Data were obtained from Ovid-MEDLINE database. 


\section{Sleep reactivity}

Sleep reactivity is the degree to which individuals exhibit acute sleep disturbance in response to stress exposure. Important to note here is that other terms used to describe sleep reactivity include "sleep system sensitivity", "stressreactivity of the sleep system", and "vulnerability to stressrelated insomnia" (in the current review, we will largely use the term "sleep reactivity" for the sake of consistency). Sleep reactivity is a ubiquitous phenomenon as all individuals experience some degree of difficulty sleeping when faced with a sufficiently potent sleep challenge. Even minor challenges, including slight changes in sleep timing and ill-timed low-dose stimulants, produce sleep disturbance in some individuals. ${ }^{42}$ This reactivity exhibits trait-like characteristics such that individuals' sleep responses to stress are consistent over time and across a variety of stressful stimuli. $^{38,42,43}$

The first efforts to distinguish between individuals with high vs low sleep reactivity relied on PSG characteristics after stress exposure (caffeine use, circadian shift, PSG adaptation night).$^{38,42}$ Individuals who had greater nocturnal wakefulness and lower sleep efficiency and yet elevated alertness on the Multiple Sleep Latency Test per PSG following laboratory stress were identified as highly reactive sleepers, whereas those whose sleep was largely unaffected by laboratory stress were characterized as having low sleep reactivity. Laboratory challenges, coupled with PSG, yield high validity for measuring sleep reactivity. Further, this approach benefits from being affectively neutral and is therefore unlikely to be contaminated by cognitive and emotional processes.

A validated assay for a priori measurement of sleep reactivity in large epidemiological samples is the Ford Insomnia Response to Stress Test (FIRST), a self-report instrument that was originally validated in a large adult sample in the USA (see Drake et al $2004^{38}$ for the full instrument). The FIRST has also been validated for use in early pregnancy ${ }^{44}$ and translated into Spanish, ${ }^{44}$ Japanese, ${ }^{45}$ European Portuguese, ${ }^{46}$ French, ${ }^{47}$ German, ${ }^{48}$ and Italian languages. ${ }^{49}$ Many studies comparing high vs low sleep reactivity in individuals have relied on using median splits to distinguish the two groups with good results. However, as medians are highly sample dependent, this has resulted in a lack of consistency regarding cutoffs across studies. To date, the sole effort to establish empirical cutoffs was based on an epidemiological sample of 2,892 good lifetime sleepers without a history of depression or insomnia, ${ }^{50}$ revealing cutoffs of FIRST $\geq 16$ reflecting moderate risk for future insomnia, and FIRST $\geq 18$ reflecting high risk for future insomnia.
A burgeoning literature supports sleep reactivity as a moderating influence of stress on the sleep system, ${ }^{38,42,51,52}$ which has drawn much attention in insomnia etiology. Despite being a normal variant, sleep reactivity offers critical insight into insomnia progression as excessive sleep reactivity yields substantial pathogenicity for insomnia development. Individuals with high sleep reactivity are vulnerable to having difficulty falling and staying asleep during and even following the removal of stress, whereas those with low sleep reactivity experience mild sleep disruptions but often return to normal without serious or persistent complications.

\section{Sleep reactivity predicts future insomnia}

Two recent prospective epidemiological studies have demonstrated high premorbid sleep reactivity as a trait-like diathesis of insomnia. In 2014, Jarrin's team assessed 1,449 lifetime good sleepers and showed that good sleepers with high sleep reactivity were at elevated risk for insomnia symptoms and chronic insomnia disorder across the following 2 years than those with low sleep reactivity. Further, evidence suggested that sleep reactivity predicted future insomnia independently of general trait hyperarousal, thus supporting sleep-specific stress reactivity as a unique factor that may be related to hyperarousal but is also a unique and independent insomnia risk factor.

In the "Evolution of Pathways to Insomnia Cohort (EPIC)" study, our team demonstrated that premorbid sleep reactivity predicted insomnia disorder incidence in a sample of 2,316 individuals without a history of Diagnostic and Statistical Manual of Mental Disorders, 4 th edition ${ }^{53}$ insomnia or major depression. ${ }^{54}$ In addition, this study offered the first evidence in support of premorbid sleep reactivity moderating the effects of major life stress on insomnia risk; the crux of earlier proposals that high sleep reactivity as an insomnia vulnerability is most pathogenic via its inability to maintain normal sleep patterns during stress. Notably, data from this sample also showed that insomniacs with high premorbid sleep reactivity are 2-3 times more likely to have "sleep onset insomnia" complaints than nonreactive sleepers at time of insomnia onset, ${ }^{50}$ with highly reactive insomniacs estimating their habitual sleep latency to be nearly twice as long as nonreactive sleepers at disease onset (65 minutes vs 37 minutes). However, symptom profiles did not differ between premorbidly high vs low sleep reactive insomniacs a year "after" onset of the disorder, indicating that phenotypic insomnia differences between high vs low sleep reactivity are most evident early in the disease course. 


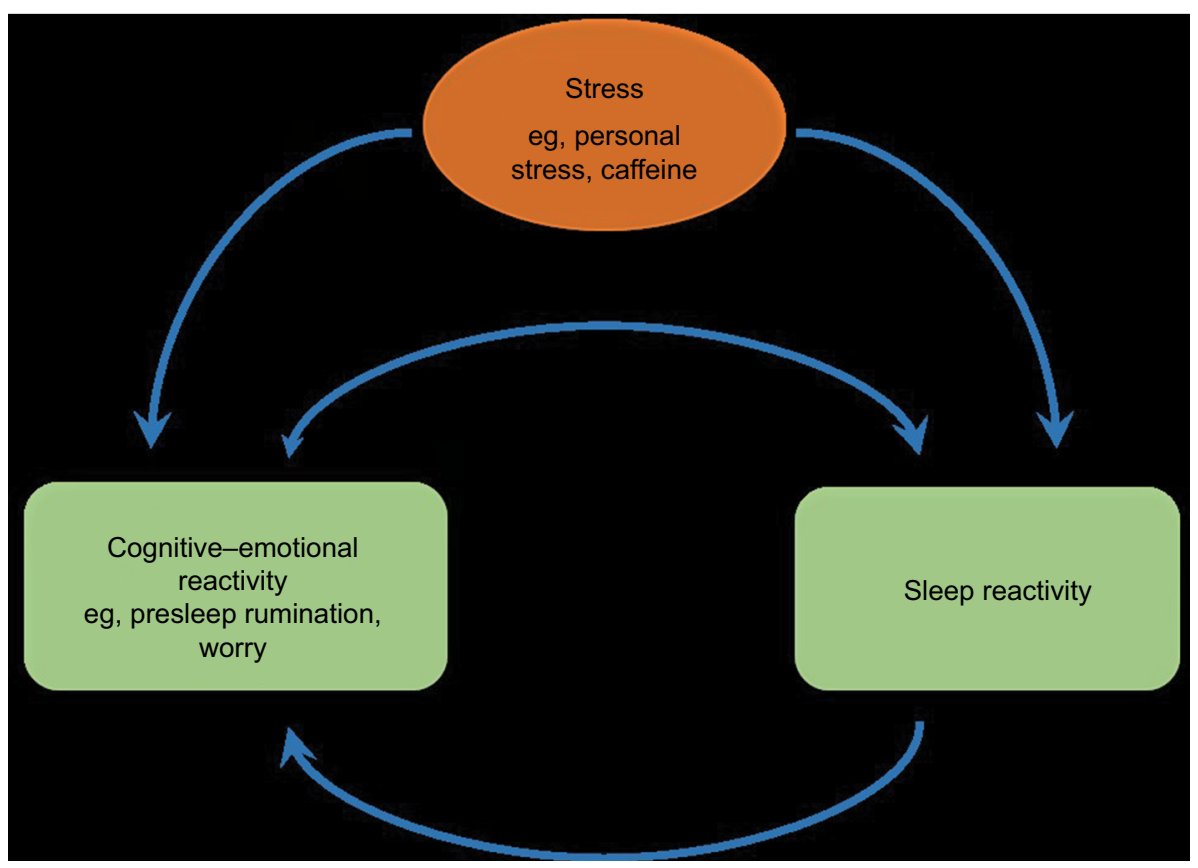

Figure 3 The bidirectional relationship, comprising of a theorized positive feedback loop, between sleep reactivity and cognitive-emotional reactivity in response to stress.

Although sleep reactivity has trait-like qualities with consistency across stressors ${ }^{38,42}$ and time, ${ }^{38,43}$ researchers have recently proposed that exposure to stress and even to insomnia itself causes changes in the regulation of stress and sleep. ${ }^{11,55,56}$ As a result, the conditions under which the initial episode of sleep disturbance develops may differ from the conditions that perpetuate its course or give rise to recurrent or chronic insomnia. Evidence from 262 subjects from the EPIC cohort showed that sleep reactivity increased substantially in insomniacs with low baseline sleep reactivity such that $68 \%$ of these insomniacs were reclassified as having high sleep reactivity; this phenomenon was dubbed "sleep system sensitization". ${ }^{57}$ In addition, exposure to major life stress augmented this sensitization process. And even after insomnia remission, sleep reactivity did not return to preinsomnia baseline levels even after disease remission, indicating that stress exposure and insomnia development have a scarring effect on the sleep system.

\section{The basis of sleep reactivity Genetics and heritability}

Consistent with evidence for a genetic basis of sleep regulation and insomnia ${ }^{55,58-63}$ and the prognosticity of familial insomnia, ${ }^{57,64-66}$ early evidence suggests a substantial polygenic influence on sleep reactivity. Twin and sibling studies estimate that $29 \%-37 \%$ of sleep reactivity is heritable, ${ }^{67,68}$ but that environmental influences play a larger role in sleep reactivity in women than in men ${ }^{69}$ Along these lines, results showed that women are substantially more sleep reactive than men, despite similar genetic architecture. Taken together, these findings suggest that greater sensitivity in the sleep system may play a critical role in increased prevalence of insomnia in women.

\section{Stress dysregulation}

An imbalance between the SNS and parasympathetic nervous system (PNS) has been implicated in cases of excessive sleep reactivity such that the SNS is hyper-active and the PNS is under-active, ${ }^{12}$ particularly when under stress, ${ }^{42}$ which is a similar pattern to what is seen between chronic insomniacs (imbalance) and good sleepers (balance). ${ }^{70}$ Building on this literature, a daily diary study showed that when individuals worried about stress and exhibited blunted PNS activity, they experienced greater stress-induced sleep disturbance, ie, higher sleep reactivity. ${ }^{71}$ This finding provides laboratory support for synergistic effects of cognitive-emotional hyperarousal and autonomic imbalance on the sleep system. However, some evidence suggests that ANS profiles may not differ between those with high vs low sleep reactivity, ${ }^{26}$ thus introducing inconsistencies into the literature that require further exploration.

In addition, HPA axis dysregulation has been posited to underlie highly reactive sleep systems, with data from the insomnia literature indicating that increased nocturnal HPA 
activity and greater HPA reactivity to stress may constitute trait-like markers for insomnia vulnerability. ${ }^{72}$ However, recent studies specifically comparing HPA responses to stress between high vs low reactive sleepers offer mixed support for exaggerated and blunted cortisol response to the same laboratory stressors. ${ }^{27,52}$ So although HPA dysregulation may contribute to stress-induced sleep disturbance, ${ }^{26}$ more research is needed to clearly understand the role of the HPA axis in sleep system reactivity.

\section{Cortical hyperarousal}

Fast electroencephalographic (EEG)-defined cortical activity during the presleep period is associated with delayed sleep onset and represents a marker of insomnia. ${ }^{73,74}$ However, the field has yet to establish cortical activity profiles associated with high sleep reactivity. Laboratory research is needed to compare cortical arousal between good sleepers with high vs low sleep reactivity. Investigating diurnal patterns, presleep profiles, and stress-induced changes would offer critical insights into potentially dysregulated cortical networks moderating the effects of stress on the sleep system.

\section{Related cognitive and emotional factors}

Explorations of the relationship between sleep reactivity and cognitive-emotional reactivity depict two independent yet related responses to stress (see Figure 2 for theoretical depiction). A large number of cognitive and emotional factors correlate with sleep reactivity, including presleep cognitive and somatic arousal, rumination, worry, perceived stress, general arousability, neuroticism, and emotion-oriented coping. ${ }^{20,71,75}$ And indeed, cognitive-emotional arousal moderates the risk for insomnia and sleep disturbance associated with sleep reactivity and stress dysregulation, ${ }^{20,71}$ thus highlighting their toxic interactions. This relationship is likely bidirectional such that when stress activates cognitive-emotional activity (eg, presleep rumination), the sensitivity of a highly reactive sleep system will be exploited, thus resulting in nighttime wakefulness and poor-quality sleep (Figure 3 ). In the opposite direction, when stress activates a highly reactive sleep system (note: the stressor can be affectively neutral ${ }^{38,42}$ or otherwise), the resultant nocturnal wakefulness creates an unstructured, typically socially isolated time in bed during which the mind is free to wander, thus representing a breeding ground for ruminative thoughts. It is possible that the interbreeding between sleep reactivity and cognitive-emotional reactivity contributes significantly to the high comorbidity between insomnia and depression. ${ }^{76,77}$

\section{Future directions}

Significant strides in insomnia research over many decades have culminated in the hyperarousal hypothesis, which has been a robust framework for the conceptualization of insomnia etiology, and targets for intervention and prevention. Sleep reactivity is a relatively young area of research but has already proved to be an integral component of the hyperarousal model. Furthermore, consideration of hyperactivity vs hyperreactivity lends crucial insights into disparate findings in the extant hyperarousal and stress dysregulation in insomnia literature. Thus, in the absence of stress, many individuals at risk for insomnia may not exhibit hyperarousal or abnormal stress regulation, which must be countenanced in insomnia etiology research.

Given the pathogenicity of elevated sleep reactivity, future research is needed to better elucidate the fundamental basis of and even peripheral factors influencing highly sensitive sleep systems. Improved characterization of its neurobiology and relation to cognitive and emotional processes will unveil novel pathways in the disease progression of insomnia, paving way for the development of preventive interventions. Recent evidence has already suggested that good sleepers with high sleep reactivity are especially prone to insomnia characterized by predominantly difficulty falling asleep at the time of disease onset, indicating that premorbid sleep reactivity segregates with insomnia phenotypes. Although symptom profiles between insomniacs with high vs low premorbid sleep reactivity do not differ a year after disease onset (indeed, changes in insomnia phenotypes over time is not uncommon ${ }^{78}$, that highly reactive sleepers primarily struggle to initiate sleep early in the course of disease offers important insights into the unique etiological factors contributing to sleep onset insomnia. Expanding on this literature, insomnia disorder with objective short sleep duration has been identified as the most treatment-resistant and devastating phenotype of insomnia in terms of morbidity and early mortality. Short sleep insomnia is characterized by severe biological hyperarousal and stress dysregulation at its foundation. Research is needed to determine whether sleep reactivity can identify those at future risk for the short sleep insomnia phenotype, as reliable premorbid predictors of this form of insomnia have not yet been established.

Last, the clinical utility of sleep reactivity as measured by the FIRST has yet to be explored. Measuring sleep reactivity in patients presenting with insomnia complaints may have value in directing to different levels of intervention. Stepped-care approaches have been recommended for cognitive-behavioral 
therapy for insomnia (CBTI), ${ }^{79}$ and early evidence supports the scalability of CBTI ${ }^{80}$ Insomniacs with low sleep reactivity may have less severe forms of insomnia trajectories and require less intensive treatment modalities such as self-guided digital/internet-based delivery of CBTI ${ }^{81-86}$ Insomniacs with severely high sleep reactivity scores, however, may require more intensive and personalized cognitive-behavioral and pharmacological treatment provided by expertly trained sleep psychologists and physicians. Future research is needed to evaluate whether sleep reactivity is associated with response to pharmacologic or cognitive-behavioral interventions for insomnia. Notably, the clinical utility of sleep reactivity may extend beyond pretreatment evaluation and may be useful in identifying remitters who may be at elevated risk for future relapse. Even after insomnia remission and the restoration of good sleep, sleep reactivity typically remains in the elevated range, suggestive of high risk for future insomnia even when asymptomatic. ${ }^{57}$ Therefore, even after treatment success, remitted insomniacs who continue to exhibit elevated levels of sleep reactivity may reduce risk for relapse by adopting ongoing maintenance treatment.

\section{Disclosure}

Dr CL Drake has received research support from Merck \& Co., Eisai Co., Aladdin Dreamer, Jazz, Actelion, and Teva and has served on speakers' bureau for Merck \& Co. Dr DA Kalmbach has received research support from Merck \& Co. The authors report no other conflicts of interest in this work.

\section{References}

1. Roth T, Coulouvrat C, Hajak G, et al. Prevalence and perceived health associated with insomnia based on DSM-IV-TR; International Statistical Classification of Diseases and Related Health Problems, Tenth Revision; and Research Diagnostic Criteria/International Classification of Sleep Disorders, Second Edition Criteria: results from the America Insomnia Survey. Biol Psychiatry. 2011;69(6):592-600.

2. Morin CM, LeBlanc M, Daley M, Gregoire J, Merette C. Epidemiology of insomnia: prevalence, self-help treatments, consultations, and determinants of help-seeking behaviors. Sleep Med. 2006;7(2):123-130.

3. Drake CL, Roehrs T, Roth T. Insomnia causes, consequences, and therapeutics: an overview. Depress Anxiety. 2003;18(4):163-176.

4. Okajima I, Komada Y, Inoue Y. A meta-analysis on the treatment effectiveness of cognitive behavioral therapy for primary insomnia. Sleep Biol Rhythms. 2011;9(1):24-34.

5. Koffel EA, Koffel JB, Gehrman PR. A meta-analysis of group cognitive behavioral therapy for insomnia. Sleep Med Rev. 2015;19:6-16.

6. Smith MT, Perlis ML, Park A, et al. Comparative meta-analysis of pharmacotherapy and behavior therapy for persistent insomnia. Am J Psychiatry. 2002;159(1):5-11.

7. Riemann D, Perlis ML. The treatments of chronic insomnia: a review of benzodiazepine receptor agonists and psychological and behavioral therapies. Sleep Med Rev. 2009;13(3):205-214.

8. Roehrs T, Roth T. Insomnia pharmacotherapy. Neurotherapeutics. 2012;9(4):728-738.
9. Morin CM, Bélanger L, LeBlanc M, et al. The natural history of insomnia: a population-based 3-year longitudinal study. Arch Intern Med. 2009;169(5):447-453.

10. Bathgate CJ, Edinger JD, Krystal AD. Insomnia patients with objective short sleep duration have a blunted response to cognitive behavioral therapy for insomnia. Sleep. 2017;40(1).

11. Riemann D, Spiegelhalder K, Feige B, et al. The hyperarousal model of insomnia: a review of the concept and its evidence. Sleep Med Rev. 2010;14(1):19-31.

12. Bonnet MH, Arand DL. Hyperarousal and insomnia: state of the science. Sleep Med Rev. 2010;14(1):9-15.

13. Roth T, Roehrs T, Pies R. Insomnia: pathophysiology and implications for treatment. Sleep Med Rev. 2007;11(1):71-79.

14. Harvey C-J, Gehrman P, Espie CA. Who is predisposed to insomnia: a review of familial aggregation, stress-reactivity, personality and coping style. Sleep Med Rev. 2014;18(3):237-247.

15. Kay DB, Buysse DJ. Hyperarousal and beyond: new insights to the pathophysiology of insomnia disorder through functional neuroimaging studies. Brain Sci. 2017;7(3):23.

16. Drummond SP, Smith MT, Orff HJ, Chengazi V, Perlis ML. Functional imaging of the sleeping brain: review of findings and implications for the study of insomnia. Sleep Med Rev. 2004;8(3):227-242.

17. Vgontzas AN, Fernandez-Mendoza J, Liao D, Bixler EO. Insomnia with objective short sleep duration: the most biologically severe phenotype of the disorder. Sleep Med Rev. 2013;17(4):241-254.

18. Healey ES, Kales A, Monroe LJ, Bixler EO, Chamberlin K, Soldatos CR. Onset of insomnia: role of life-stress events. Psychosom Med. 1981;43(5):439-451.

19. Morin CM, Rodrigue S, Ivers H. Role of stress, arousal, and coping skills in primary insomnia. Psychosom Med. 2003;65(2):259-267.

20. Drake CL, Pillai V, Roth T. Stress and sleep reactivity: a prospective investigation of the stress-diathesis model of insomnia. Sleep. 2014;37(8): 1295-1304.

21. Xia L, Chen G-H, Li Z-H, Jiang S, Shen J. Alterations in hypothalamuspituitary-adrenal/thyroid axes and gonadotropin-releasing hormone in the patients with primary insomnia: a clinical research. PloS One. 2013;8(8):e71065.

22. Riemann D, Klein T, Rodenbeck A, et al. Nocturnal cortisol and melatonin secretion in primary insomnia. Psychiatry Res. 2002;113(1):17-27.

23. Gehrman PR, Hall M, Barilla H, et al. Stress reactivity in insomnia. Behav Sleep Med. 2016;14(1):23-33.

24. Mikoteit T, Pawlowski M, Seiffert N, et al. Heart rate variability during sleep distinguishes between insomnia and normal sleep. Sleep Med. 2017;40:e223-e224

25. Dodds KL, Miller CB, Kyle SD, Marshall NS, Gordon CJ. Heart rate variability in insomnia patients: a critical review of the literature. Sleep Med Rev. 2017;33:88-100.

26. Chen IY, Jarrin DC, Ivers H, Morin CM. Investigating psychological and physiological responses to the Trier Social Stress Test in young adults with insomnia. Sleep Med. 2017;40:11-22.

27. Drake CL, Cheng P, Almeida DM, Roth T. Familial risk for insomnia is associated with abnormal cortisol response to stress. Sleep. 2017;40(10).

28. Vgontzas AN, Bixler EO, Lin H-M, et al. Chronic insomnia is associated with nyctohemeral activation of the hypothalamic-pituitary-adrenal axis: clinical implications. J Clin Endocrinol Metab. 2001;86(8):3787-3794.

29. Nofzinger EA, Buysse DJ, Germain A, Price JC, Miewald JM, Kupfer DJ. Functional neuroimaging evidence for hyperarousal in insomnia. Am J Psychiatry. 2004;161(11):2126-2128.

30. Fernandez-Mendoza J, Li Y, Vgontzas AN, et al. Insomnia is associated with cortical hyperarousal as early as adolescence. Sleep. 2016;39(5):1029-1036.

31. Wołyńczyk-Gmaj D, Szelenberger W. Waking EEG in primary insomnia. Acta Neurobiol Exp. 2011;71:387-392.

32. Smith MT, Perlis ML, Chengazi VU, et al. Neuroimaging of NREM sleep in primary insomnia: a Tc-99-HMPAO single photon emission computed tomography study. Sleep. 2002;25(3):325-335. 
33. Spiegelhalder K, Regen W, Baglioni C, Riemann D, Winkelman JW. Neuroimaging studies in insomnia. Curr Psychiatry Rep. 2013;15(11): 405.

34. Ellis BJ, Jackson JJ, Boyce WT. The stress response systems: universality and adaptive individual differences. Dev Rev. 2006;26(2):175-212.

35. Selye H. The nature of stress. Basal Facts. 1985;7(1):3-11.

36. Pacak K, Palkovits M. Stressor specificity of central neuroendocrine responses: implications for stress-related disorders. Endocr Rev. 2001;22(4):502-548.

37. Kobasa SC, Maddi SR, Courington S. Personality and constitution as mediators in the stress-illness relationship. J Health Soc Behav. 1981:368-378.

38. Drake C, Richardson G, Roehrs T, Scofield H, Roth T. Vulnerability to stress-related sleep disturbance and hyperarousal. Sleep. 2004;27(2): 285-291.

39. Drake CL, Roth T. Predisposition in the evolution of insomnia: evidence, potential mechanisms, and future directions. Sleep Med Clin. 2006;1(3):333-349.

40. Fernández-Mendoza J, Vela-Bueno A, Vgontzas AN, et al. Cognitiveemotional hyperarousal as a premorbid characteristic of individuals vulnerable to insomnia. Psychosom Med. 2010;72(4):397-403.

41. Baglioni C, Spiegelhalder K, Lombardo C, Riemann D. Sleep and emotions: a focus on insomnia. Sleep Med Rev. 2010;14(4):227-238.

42. Bonnet MH, Arand DL. Situational insomnia: consistency, predictors, and outcomes. Sleep. 2003;26(8):1029-1036.

43. Jarrin DC, Chen IY, Ivers H, Drake CL, Morin CM. Temporal stability of the ford insomnia response to stress test (first). J Clin Sleep Med. 2016;12(10): 1373 .

44. Gelaye B, Zhong Q-Y, Barrios YV, Redline S, Drake CL, Williams MA. Psychometric evaluation of the Ford Insomnia Response to Stress Test (FIRST) in early pregnancy. J Clin Sleep Med. 2016;12(4):579-587.

45. Nakajima S, Okajima I, Sasai T, et al. Validation of the Japanese version of the Ford Insomnia Response to Stress Test and the association of sleep reactivity with trait anxiety and insomnia. Sleep Med. 2014;15(2): 196-202.

46. Marques DR, Allen Gomes A, Drake CL, Roth T, de Azevedo MHP. Assessing stress-induced sleep reactivity in college students: the European Portuguese version of the Ford insomnia response to stress test (FIRST). Behav Sleep Med. 2016;16(4):1-12.

47. Chen I, Jarrin D, Rochefort A, Lamy M, Ivers H, Morin C. Validation of the French version of the Ford insomnia response to stress test and the association between sleep reactivity and hyperarousal. Sleep Med. 2015;16(1):S238.

48. Dieck A, Helbig S, Drake CL, Backhaus J. Validation of the German version of the Ford Insomnia Response to Stress Test. J Sleep Res. Epub 2017 Oct 19.

49. Palagini L, Bruno RM, Paolo T, et al. Association between stress-related sleep reactivity and metacognitive beliefs about sleep in insomnia disorder: preliminary results. Behav Sleep Med. 2016;14(6):636-649.

50. Kalmbach DA, Pillai V, Arnedt JT, Drake CL. Identifying at-risk individuals for insomnia using the Ford Insomnia Response to Stress Test. Sleep. 2016;39(2):449-456.

51. Petersen H, Kecklund G, D’onofrio P, Nilsson J, Åkerstedt T. Stress vulnerability and the effects of moderate daily stress on sleep polysomnography and subjective sleepiness. J Sleep Res. 2013;22(1):50-57.

52. Chen I, Jarrin D, Ivers H, Rochefort A, Morin C. Association between stress-induced arousal and nocturnal sleep: a preliminary study. Sleep Med. 2017;40:e55.

53. American Psychiatric Association. Diagnostic and Statistical ManualText Revision (DSM-IV-TR, 2000). Washington DC: American Psychiatric Association; 2000.

54. Drake CL, Pillai V, Roth T. Stress and sleep reactivity: a prospective investigation of the stress-diathesis model of insomnia. Sleep. 2013;37(8):1295-1304.

55. Palagini L, Biber K, Riemann D. The genetics of insomnia - evidence for epigenetic mechanisms? Sleep Med Rev. 2014;18(3):225-235.
56. Meerlo P, Sgoifo A, Suchecki D. Restricted and disrupted sleep: effects on autonomic function, neuroendocrine stress systems and stress responsivity. Sleep Med Rev. 2008;12(3):197-210.

57. Kalmbach DA, Pillai V, Arnedt JT, Anderson JR, Drake CL. Sleep system sensitization: evidence for changing roles of etiological factors in insomnia. Sleep Med. 2016;21:63-69.

58. Gehrman PR, Byrne E, Gillespie N, Martin NG. Genetics of insomnia. Sleep Med Clin Rev. 2011(6):191-202.

59. Gehrman PR, Meltzer LJ, Moore M, et al. Heritability of insomnia symptoms in youth and their relationship to depression and anxiety. Sleep. 2011;34(12):1641.

60. Lind MJ, Gehrman PR. Genetic pathways to insomnia. Brain Sci. 2016;6(4):64.

61. Lane JM, Liang J, Vlasac I, et al. Genome-wide association analyses of sleep disturbance traits identify new loci and highlight shared genetics with neuropsychiatric and metabolic traits. Nat Genet. 2017;49(2):274.

62. Hammerschlag AR, Stringer S, de Leeuw CA, et al. Genome-wide association analysis of insomnia complaints identifies risk genes and genetic overlap with psychiatric and metabolic traits. Nature Genetics. 2017;49(11):1584-1592.

63. Pack AI, Keenan BT, Byrne EM, Gehrman PR. Genetics and genomic basis of sleep disorders in humans. In: Kryger MH, Roth T, Dement WC, editors. Principles and Practice of Sleep Medicine. 6th ed. St. Louis (MO): Elsevier; 2016.

64. Singareddy R, Vgontzas AN, Fernandez-Mendoza J, et al. Risk factors for incident chronic insomnia: a general population prospective study. Sleep Med. 2012;13(4):346-353.

65. Bastien CH, Morin CM. Familial incidence of insomnia. J Sleep Res. 2000;9(1):49-54.

66. Dauvilliers Y, Morin C, Cervena K, et al. Family studies in insomnia. J Psychosom Res. 2005;58(3):271-278.

67. Drake CL, Scofield H, Roth T. Vulnerability to insomnia: the role of familial aggregation. Sleep Med. 2008;9(3):297-302.

68. Fernandez-Mendoza J, Shaffer ML, Olavarrieta-Bernardino S, et al. Cognitive-emotional hyperarousal in the offspring of parents vulnerable to insomnia: a nuclear family study. J Sleep Res. 2014;23(5): 489-498.

69. Drake CL, Friedman NP, Wright KP, Jr, Roth T. Sleep reactivity and insomnia: genetic and environmental influences. Sleep. 2011;34(9): 1179-1188.

70. Bonnet M, Arand D. Heart rate variability in insomniacs and matched normal sleepers. Psychosom Med. 1998;60(5):610-615.

71. Gouin J, Wenzel K, Deschenes S, Dang-Vu T. Heart rate variability predicts sleep efficiency. Sleep Med. 2013;14:e142.

72. Riemann D, Spiegelhalder K, Feige B, et al. The hyperarousal model of insomnia: a review of the concept and its evidence. Sleep Med Rev. 2010;14(1):19-31.

73. Perlis ML, Merica H, Smith MT, Giles DE. Beta EEG activity and insomnia. Sleep Med Rev. 2001;5(5):365-376.

74. Fernandez-Mendoza J, Li Y, Vgontzas A, et al. Insomnia is associated with cortical hyperarousal as early as adolescence. Sleep. 2016;39(5): 1029-1036.

75. Fernandez-Mendoza J, Vela-Bueno A, Vgontzas AN, et al. Cognitiveemotional hyperarousal as a premorbid characteristic of individuals vulnerable to insomnia. Psychosom Med. 2010;72(4):397-403.

76. Riemann D, Voderholzer U. Primary insomnia: a risk factor to develop depression? J Affect Disord. 2003;76(1):255-259.

77. Tsuno N, Besset A, Ritchie K. Sleep and depression. J Clin Psychiatry. 2005;66:1254-1269.

78. Pillai V, Roth T, Drake CL. The nature of stable insomnia phenotypes. Sleep. 2015;38(1):127-138.

79. Espie CA. "Stepped care": a health technology solution for delivering cognitive behavioral therapy as a first line insomnia treatment. Sleep. 2009;32(12):1549-1558.

80. Manber R, Simpson NS, Bootzin RR. A step towards stepped care: delivery of CBT-I with reduced clinician time. Sleep Med Rev. 2015;19:3-5. 
81. Zachariae R, Lyby MS, Ritterband LM, O'Toole MS. Efficacy of internet-delivered cognitive-behavioral therapy for insomnia - a systematic review and meta-analysis of randomized controlled trials. Sleep Med Rev. 2016;30:1-10.

82. Espie CA, Hames P, McKinstry B. Use of the internet and mobile media for delivery of cognitive behavioral insomnia therapy. Sleep Med Clin. 2013;8(3):407-419.

83. Feuerstein S, Hodges SE, Keenaghan B, Bessette A, Forselius E, Morgan PT. Computerized cognitive behavioral therapy for insomnia in a community health setting. J Clin Sleep Med. 2017;13(2):267-274.
84. Pillai V, Anderson JR, Cheng P, et al. The anxiolytic effects of cognitive behavior therapy for insomnia: preliminary results from a web-delivered protocol. J Sleep Med Disord. 2015;2(2):a-7.

85. Lancee J, van Straten A, Morina N, Kaldo V, Kamphuis JH. Guided online or face-to-face cognitive behavioral treatment for insomnia: a randomized wait-list controlled trial. Sleep. 2016;39(1):183-191.

86. Blom K, Tillgren HT, Wiklund T, et al. Internet-vs. group-delivered cognitive behavior therapy for insomnia: a randomized controlled noninferiority trial. Behav Res Ther. 2015;70:47-55.

\section{Publish your work in this journal}

Nature and Science of Sleep is an international, peer-reviewed, open access journal covering all aspects of sleep science and sleep medicine, including the neurophysiology and functions of sleep, the genetics of sleep, sleep and society, biological rhythms, dreaming, sleep disorders and therapy, and strategies to optimize healthy sleep. The manuscript

\section{Dovepress}

management system is completely online and includes a very quick and fair peer-review system, which is all easy to use. Visit http://www. dovepress.com/testimonials.php to read real quotes from published authors. 